\title{
Enhancement of the Ultraviolet Luminescence Intensity from Cd-Doped ZnO Films Caused by Exciton Binding
}

\author{
I. Shtepliuk ${ }^{a, *}$, G. Lashikarev ${ }^{a}$, O. Khyzhun ${ }^{a}$, B. Kowalski ${ }^{b}$, A. Reszka ${ }^{b}$, V. Khomyak ${ }^{c}$, \\ V. LAZORENKO ${ }^{a}$ AND I. TIMOFEEVA ${ }^{a}$ \\ ${ }^{a}$ I. Frantsevich Institute for Problems of Material Science, NASU, 03680, Kiev-142, Ukraine \\ ${ }^{b}$ Institute of Physics, Polish Academy of Sciences, Warsaw, Poland \\ ${ }^{c}$ Chernivtsi National University, 58012, Chernivtsi, Ukraine
}

\begin{abstract}
$\mathrm{ZnO}$ films doped with the cadmium (0.4-0.6\%) were grown on crystalline sapphire c- $\mathrm{Al}_{2} \mathrm{O}_{3}$ substrates applying radiofrequency magnetron sputtering at the temperature of $400{ }^{\circ} \mathrm{C}$ in $\mathrm{Ar}-\mathrm{O}_{2}$ atmosphere. The as-grown films were investigated in detail using X-ray diffraction, X-ray photoelectron spectroscopy, and cathodoluminescence spectra. The X-ray diffraction analysis revealed that the films possess a hexagonal wurtzite-type structure with the dominant crystallite orientation along the $c$ axis. It was found that the small concentration of the cadmium significantly enhances the ultraviolet emission associated with excitonic transitions. We suggest that this enhancement effect mainly results from appearance of the cadmium isoelectronic traps, which may bind an exciton, thereby increasing the probability of radiation recombination. The effect of Cd isoelectronic impurity on structural and luminescent properties of $\mathrm{ZnO}$ films is discussed.
\end{abstract}

PACS: 78.55.Et, 78.67.Bf, 81.05.Dz, 81.07.Bc

\section{Introduction}

Recently, zinc oxide ( $\mathrm{ZnO})$ films have been extensively investigated due to their potential for the development of novel optoelectronic devices. Their excellent properties such as a wide band gap $(3.37 \mathrm{eV}$ at $300 \mathrm{~K})$, a large exciton binding energy $(60 \mathrm{meV})$, and a large melting point of $1975{ }^{\circ} \mathrm{C}$ ensure applications in photodetectors, light emitting diodes and laser light source [1-3]. For $\mathrm{ZnO}$, addition of impurities often induces dramatic changes in their electrical and optical properties, which suggests new applications.

For the development of devices based on the zinc oxide, the knowledge about the effect of isoelectronic impurities is of essential interest. It is known that the isoelectronic traps can improve the efficiency of radiative recombination of electrons and holes, as was shown for the light-emitting diodes based on GaP:N [4]. In the paper [5], the authors report the observation of an exciton bound with isoelectronic nitrogen atom in GaP. According to Hopfield et al., the difference of electronegativity between nitrogen and phosphor was suggested to be responsible for the binding of the exciton to the nitrogen center [6]. Alternatively, the binding mechanism can be described as a consequence of the lattice deformation due to the atomic size difference between impurity and host atom.

The goal of this paper is to determine the degree of the cadmium doping influence on the ultraviolet emission of zinc oxide films. In our work it is shown that the isoelectronic impurity of cadmium (0.4 at.\%) leads to improvement in structural properties and cathodolu-

* corresponding author; e-mail: shtepliuk_1987@ukr.net minescence of zinc oxide films. By analogy with GaP:N, we explain the enhancement of the UV emission intensity by the appearance of cadmium isoelectronic traps which can bind an exciton. This increases the probability of radiative recombination, i.e. luminescence intensity.

\section{Experimental details}

$\mathrm{ZnO}$ films doped with cadmium were deposited on c- $\mathrm{Al}_{2} \mathrm{O}_{3}$ substrates at the temperature $400^{\circ} \mathrm{C}$ by the $\mathrm{RF}$ magnetron sputtering. Targets with different ratios of $\mathrm{Zn}$ and $\mathrm{Cd}$ have a form of zinc metal discs with cadmium inserts $(2,4,6)$. Vacuum in deposition chamber was $10^{-3} \mathrm{~Pa}$. The mixture of high-purity (99.99\%) argon and oxygen were used as working gases. The specific flux of argon and oxygen was maintained at a ratio of $\mathrm{Ar}: \mathrm{O}_{2} \approx 2: 1$. The distance from the target to the substrate was fixed at $40 \mathrm{~mm}$. The discharge power was $200 \mathrm{~W}$. In order to obtain perfect films, layer-by-layer deposition method was used $[7,8]$. The deposition time was 60 min for all samples. The growth process was carried out in four stages. After deposition of every layer the deposition process was interrupted. The interruption time is $5 \mathrm{~min}$. Before deposition of each film, the zinc-cadmium target was presputtered in $\mathrm{Ar}-\mathrm{O}_{2}$ atmosphere for about $10 \mathrm{~min}$ in order to remove the surface oxide. The growth rate is about $30 \mathrm{~nm} / \mathrm{min}$. The crystal structure of samples was studied by the X-ray diffraction (XRD) where $\mathrm{Cu} K_{\alpha}$ radiation was used as a source $(\lambda=0.154056 \mathrm{~nm})$. The XRD measurements were carried out with the DRON-4 system. X-ray photoelectron spectroscopy (XPS) valence-band and core-level spectra were measured using the UHV-Analysis-System assembled by SPECS (Germany). The system is equipped with 
a PHOIBOS 150 hemispherical analyzer. A base pressure in the sublimation ion-pumped chamber of the system was less than $9 \times 10^{-8} \mathrm{~Pa}$ during the present experiments. The $\mathrm{Mg} K_{\alpha}$ radiation $(E=1253.6 \mathrm{eV})$ was used as a source of XPS spectra excitation. The XPS spectra were measured at the constant pass energy of $25 \mathrm{eV}$. The binding energy (BE) of $84.00 \pm 0.05 \mathrm{eV}$ of the XPS $\mathrm{Au}$ $4 f_{7 / 2}$ core-level spectrum was used as a reference. Energy drift due to charging effects was calibrated, taking the XPS C $1 s(285.0 \mathrm{eV})$ core-level spectrum of hydrocarbons. The cathodoluminescence (CL) spectra were taken in a JEOL $35 \mathrm{C}$ scanning electron microscope equipped with an Oxford Instruments MonoCL2 CL system and Hamamatsu R943-02 Peltier cooled photomultiplier. All CL spectra discussed here were taken at room temperature.

\section{Results and discussions}

We have obtained the undoped and Cd-doped $\mathrm{ZnO}$ films by the rf magnetron sputtering technique. In order to determine the atomic concentrations of the cadmium in the $\mathrm{Cd}$-doped $\mathrm{ZnO}$ films, we investigated the XPS spectra for these samples (Fig. 1a). The Cd content in the $\mathrm{ZnO}$ films can be evaluated by comparing the integrated areas of the $\mathrm{Cd} 3 d_{5 / 2}$ peak and the sum of the $\mathrm{Cd}$ $3 d_{5 / 2}$ peak together with the $\mathrm{Zn} 2 p_{3 / 2}$ peak. Considering their ionization cross-sections, the cadmium content for our films is a small one about $0.4 \%, 0.5 \%$, and $0.6 \%$, respectively (Fig. 1b).

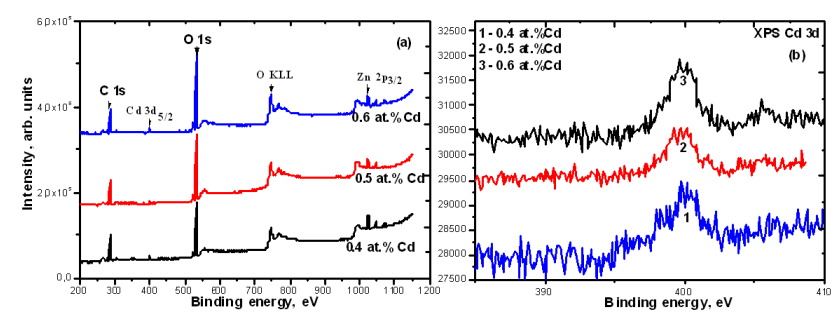

Fig. 1. XPS spectra of Cd-doped (a) and XPS Cd $3 d$ core-levels spectra (b) of $\mathrm{ZnO}$ films deposited on the c- $\mathrm{Al}_{2} \mathrm{O}_{3}$ substrates.

Figure $2 \mathrm{a}$ shows the XRD patterns of undoped and $\mathrm{Cd}$-doped $\mathrm{ZnO}$ films, in which two prominent peaks are observed. These two peaks are indexed to be (002), (004) crystal planes of hexagonal $\mathrm{ZnO}$ according to the standard JCPDS Card (No. 36-1451).

With increasing $\mathrm{Cd}$ content, a small decrease of the diffraction angle was observed (Fig. 2b), indicating that the larger ion $\mathrm{Cd}^{2+}$ (ionic radius $0.97 \AA$ ) replaces the smaller ion $\mathrm{Zn}^{2+}$ (ionic radius $0.74 \AA$ ) in the cationic sublattice of $\mathrm{ZnO}$. The values of the full width at half maximum (FWHM) for the crystallographic plane (002) are also presented in Fig. 2b. When the cadmium content is about 0.4 at.\%, the FWHM reaches the lowest value, indicating the better structural quality of the 0.4 at.\% Cd-doped films.

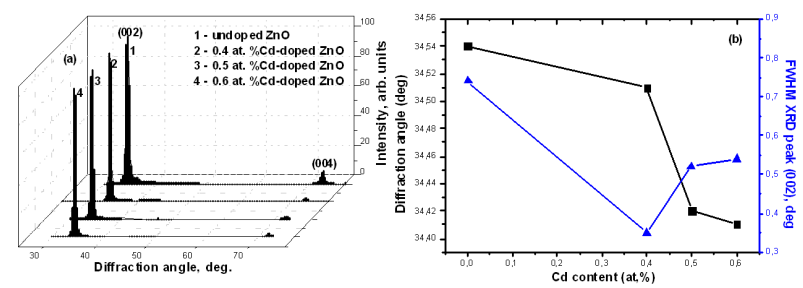

Fig. 2. XRD patterns (a) for undoped and Cd-doped $\mathrm{ZnO}$ films deposited on the c- $\mathrm{Al}_{2} \mathrm{O}_{3}$ substrates as well as dependences of the diffraction angle ( $\boldsymbol{\square})$ and the FWHM peak $(\boldsymbol{\Lambda})$ on the cadmium content (b).

Figure 3 shows the CL spectra measured at electron beam energy of $15 \mathrm{keV}$. For $0.4 \% \mathrm{Cd}$-doped $\mathrm{ZnO}$ film a strong UV near-band emission at approximately $382 \mathrm{~nm}$ related to the excitonic transition is observed. It can be seen that CL intensity for the sample with 0.4 at.\% cadmium is 1.5 times higher than its value for undoped $\mathrm{ZnO}$ (Fig. 4a). The enhancement of luminescence intensity is due to increasing the probability of the processes of radiative recombination. It can be due to the fact that the isoelectronic cadmium atom in $\mathrm{ZnO}$ is able to bind an exciton. In order to explain the binding of the exciton to the isoelectronic $\mathrm{Cd}$ atom in $\mathrm{ZnO}$, the model of Hopfield et al. [6] might be applied. Hopfield et al. assumed that an isoelectronic trap could be attractive for either electrons or holes. Since there is no net charge involved, the binding will not be due to the Coulomb forces but to forces of very short range. Therefore, it will be impossible to treat this bound state with an effective mass approximation.

After an isoelectronic trap has captured an electron or a hole, this trap is then negatively or positively charged. By the Coulomb interaction, it will capture a carrier of opposite charge. This leads to an exciton bound to the isoelectronic trap. The Pauling electronegativities of $\mathrm{Zn}$ and $\mathrm{Cd}$ are 1.65 and 1.70, respectively. Thus, within this model, Cd tends to bind an electron more strongly than Zn. Then via the Coulomb interaction an electron binds a hole and, thereby, forms an exciton. In addition, covalent radii of zinc and cadmium are $0.131 \mathrm{~nm}$ and $0.148 \mathrm{~nm}$, respectively, i.e. the covalent radius of $\mathrm{Cd}$ is $13 \%$ greater than the covalent radius of zinc. Due to the induced lattice deformation about the $\mathrm{Cd}$ atoms a strain field should develop. Both mechanisms can explain the formation of an isoelectronic trap at $\mathrm{Cd}$ in $\mathrm{ZnO}$ in a qualitative way.

It was also found from CL spectra that the peak of near band (NBE) emission of $0.4 \% \mathrm{Cd}$-doped $\mathrm{ZnO}$ film, in comparison to undoped $\mathrm{ZnO}$, blue shifted to the higher-energy side by $17 \mathrm{meV}$; and the red shift for $0.5 \% \mathrm{Cd}$ -doped and $0.6 \% \mathrm{Cd}$-doped $\mathrm{ZnO}$ films was about $21 \mathrm{meV}$ and $28 \mathrm{meV}$, respectively (Fig. 4b).

Simple diagram of the blue shifting of the NBE emission of $0.4 \% \mathrm{Cd}$-doped $\mathrm{ZnO}$ film is presented in Fig. 5 . The blue shift of NBE emission in the $0.4 \% \mathrm{Cd}$-doped 


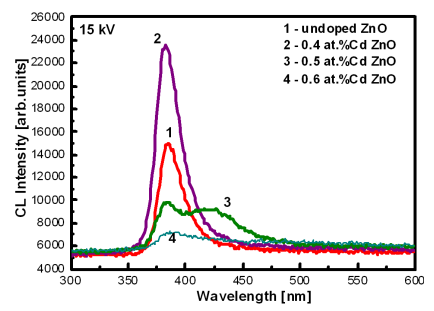

Fig. 3. CL spectra for undoped and Cd-doped $\mathrm{ZnO}$ films deposited on the $\mathrm{c}-\mathrm{Al}_{2} \mathrm{O}_{3}$ substrates at electron energy of $15 \mathrm{keV}$.

$\mathrm{ZnO}$ film is believed to be caused by the Burstein-Moss effect [9]. It is well known that group (II) element dopants, such as $\mathrm{Cd}$, act as donors in $\mathrm{ZnO}$. The donor electrons occupy states at the bottom of the conduction band. Since the Pauli principle prevents double occupancy of states and optical transitions are vertical, the low-energy transitions are blocked. Blockage of the low-energy transitions is known as the Burstein-Moss effect, and it enhances optical energy.
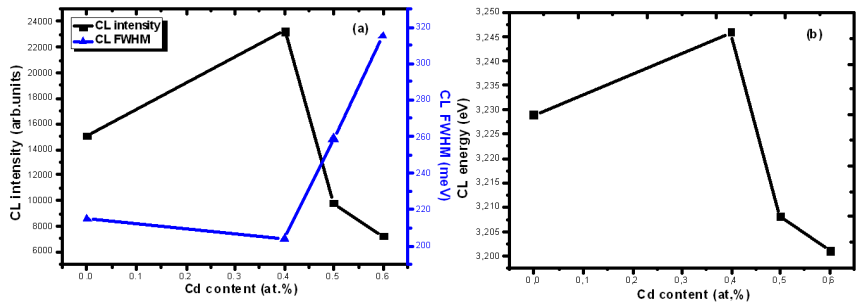

Fig. 4. Dependences of the CL intensity, CL FWHM (a) and CL energy (b) on the cadmium content.
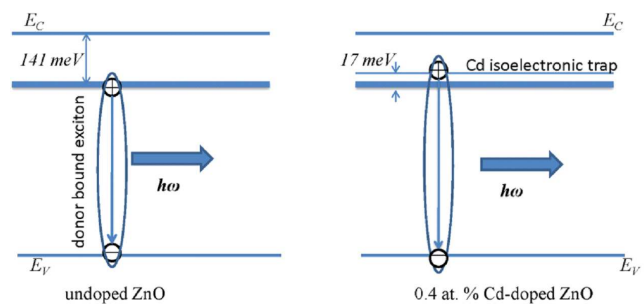

Fig. 5. A simple diagram showing the excitonic transitions in the undoped and 0.4 at.\% Cd-doped $\mathrm{ZnO}$ films.

The red shift of $\mathrm{NBE}$ emission in $0.5 \% \mathrm{Cd}$-doped and $0.6 \% \mathrm{Cd}$-doped $\mathrm{ZnO}$ films cannot be attributed to electrons filling in the lower part of the conduction band, as in the case of the Burstein-Moss effect. This indicates that the band structure, including the band gap itself, must have been substantially modulated. It was found that the CL intensity of $0.5 \% \mathrm{Cd}$-doped and $0.6 \% \mathrm{Cd}-$ -doped $\mathrm{ZnO}$ films significantly decreases compared to the $0.4 \% \mathrm{Cd}$-doped $\mathrm{ZnO}$ film, while the $\mathrm{CL}$ linewidth (FWHM) increases (Fig. 4a). This increase of broadening of $0.5 \% \mathrm{Cd}$-doped and $0.6 \% \mathrm{Cd}$-doped $\mathrm{ZnO}$ films is mainly due to the increase of disorder in semiconductor, which leads to the appearance of localized electron and/or hole states [10]. Two localization mechanisms have been suggested [11]. One is that exciton is localized by the Coulomb interaction in very deep potential wells. The other is a case of wide potential well with dimensions larger than the excitonic Bohr radius. In this case, the exciton is localized as a whole in a wide potential well. Martin et al. [10] suggested the additional mechanism. An additional localizing mechanism may be found in the nature of the segregated quantum dots (confinement effect) or alternatively from the large piezoelectric fields present in wurtzite semiconductors (the Coulomb effect). Emission spectra are broadened due to these localized states. Nevertheless, the proposed interpretations of the red- and blue shift of the NBE emission are tentative and need further verification.

\section{Conclusions}

The Cd-doped $\mathrm{ZnO}$ films with different cadmium concentrations have been prepared by radiofrequency magnetron sputtering method on sapphire substrates at $400{ }^{\circ} \mathrm{C}$. The films were characterized by different methods to understand their structural, optical and chemical properties. The X-ray diffraction analysis revealed that the films are polycrystalline in nature having a hexagonal wurtzite type crystal structure with a preferred grain orientation in the (002) direction. It was found that the small concentration of the cadmium (0.4 at.\%) significantly enhances the ultraviolet emission associated with excitonic transitions. We suggest that this enhancement effect mainly results from appearance of the cadmium isoelectronic traps, which may bind an exciton, thereby increasing the probability of radiation recombination. CL measurements showed that the band gap decreases from 3.229 (undoped $\mathrm{ZnO}$ ) to $3.201 \mathrm{eV}(0.6$ at.\% Cd) with increasing $\mathrm{Cd}$ concentration. Increasing the $\mathrm{Cd}$ concentration ( $>0.4$ at.\%) also leads to the broadening of the emission peak and degrading the crystalline quality. The characterization studies clearly indicate the incorporation of $\mathrm{Cd}$ into $\mathrm{ZnO}$; hence the observed decrease in the CL energy which can be directly attributed to the effect of $\mathrm{Cd}$ ion incorporation into the $\mathrm{ZnO}$ lattice. The possible mechanisms of the $\mathrm{Cd}$ influence on the luminescence features of the $\mathrm{ZnO}$ films are proposed.

\section{References}

[1] O. Lupan, L. Chow, G. Chai, L. Chernyak, O. Lopatiuk-Tirpak, H. Heinrich, Phys. Status Solidi A 205, 2673 (2008).

[2] S.D. Lee, Y.S. Kim, M.S. Yi, J.Y. Choi, S.W. Kim, J. Phys. Chem. C 113, 8954 (2009).

[3] Y. Ohno, T. Shirahama, S. Takeda, A. Ishizumi, Y. Kanemitsu, Appl. Phys. Lett. 87, 043106 (2005).

[4] P.J. Dean, J. Lumin. 7, 51 (1973).

[5] D.G. Thomas, J.J. Hopfield, C.J. Frosch, Phys. Rev. Lett. 15, 857 (1965). 
[6] J.J. Hopfield, D.G. Thomas, R.T. Lynch, Phys. Rev. Lett. 17, 312 (1966).

[7] A. Ievtushenko, V. Karpyna, G. Lashkarev, V. Lazorenko, V. Baturin, A. Karpenko, M. Lunika, A. Dan'ko, Acta Phys. Pol. A 114, 1131 (2008).

[8] A.I. Ievtushenko, V.A. Karpyna, V.I. Lazorenko, G.V. Lashkarev, V.D. Khranovskyy, V.A. Baturin, O.Y. Karpenko, M.M. Lunika, K.A. Avramenko, V.V. Strelchuk, O.M. Kutsay, Thin Solid Films 518, 4529 (2010).
[9] J.I. Pankove, Optical Processes in Semiconductors, Prentice-Hall, NJ 1971.

[10] R.W. Martin, P.G. Middleton, K.P. O'Donnell, W. Van der Stricht, Appl. Phys. Lett 74, 263 (1999).

[11] C.F. Klingshirn, Semiconductor Optics, Springer, Berlin 1995. 